\title{
Data preprocessing for machine analysis of sales representatives' key performance indicators
}

\author{
Alla Yu. Vladova ${ }^{a, b}$ \\ E-mail: ayvladova@fa.ru \\ Elena D. Shek ${ }^{\mathrm{c}}$ \\ E-mail: 1399selena@gmail.com
}

\author{
${ }^{a}$ V.A. Trapeznikov Institute of Control Sciences, Russian Academy of Sciences \\ Address: 65, Profsoyuznaya Street, Moscow 117997, Russia \\ ${ }^{\mathrm{b}}$ Financial University under the Government of the Russian Federation \\ Address: 49, Leningradsky Prospect, Moscow 125993, Russia \\ ${ }^{c}$ Plekhanov Russian University of Economics \\ Address: 36, Stremyanny Lane, Moscow 117997, Russia
}

Abstract

Significant transformation of the operational activity of product and service distributors is driven by changes in data-receiving and processing technology. At present, the work of these companies' representatives is digitized to a large extent: for example, the road time, the number and places of meetings with customers are automatically recorded. At the same time, the productivity of managers who do not make direct sales is usually evaluated with the help of surveys, experts and costly double visits, although the existence of large data samples makes possible the use of statistical analysis to identify both insufficient and inflated values of performance indicators. Source data: a relational database that accumulates information about 28 categorical, quantitative, geolocation and temporal parameters of sale representatives' activities for the last year. Based on available data, we created synthetic features (the latitude and longitude features produced the index, region, street, and house features; based upon identifiers we calculated the sum of activities of sales representatives; according to temporary features we defined the season of the year, the day of the week and the period of day features). The methodology for statistical analysis consists of three main stages: collection and processing of primary data; summary and grouping processed information; setting statistical hypotheses and interpreting the results. A probabilistic approach was used to model the level of distortion of sale representatives'
} 
activities. As a result, with the built tag cloud we highlighted: the most popular season for advertising campaigns; the most productive departments and sale representatives; days of the week with the largest number of contacts to customers. We established a significant number of records about meetings with clients at the weekends. As a result of the data mining, we made a statistical hypothesis about the possibility of identifying the sale representatives who distort the number and parameters of meetings. A set of synthetic integer, real and categorical features was created to identify hidden relationships. Doubtful data (such as working at weekends or at night) were revealed. The resulting aggregated dataset is grouped by a sale representative's activity ID and the distribution of this feature is plotted. For each sale representative, integer and real features are summarized and outliers that characterize inefficient performance or distortion of data have been detected. Thus, the presence of a large sample of data on the history of movements and activities allowed us to evaluate the productivity of the distribution company's sales representatives based upon indirect features.

Key words: machine learning; key performance indicator; database; time series; geolocation; unsupervised learning; sales representative; $b 2 b$.

Citation: Vladova A.Yu., Shek E.D. (2021) Data preprocessing for machine analysis of sales representatives' key performance indicators. Business Informatics, vol. 15, no 3, pp. 48-59.

DOI: 10.17323/2587-814X.2021.3.48.59

\section{Introduction}

1 key performance indicator (KPI) system is most effective in large companies, where it is more difficult to distinguish the contribution of each employee compared to small enterprises. The main positive aspect of KPI implementation is the ability to quantify employee activity and followup planning. And one of the negative aspects is that in the absence of control, an employee adapts his performance to the requirements of the KPI.

The daily work of a sales representative (SR) within the framework of a product or service promotional campaign consists of several meetings (activities) with customers when he $\backslash$ she demonstrates promotional material in special software. This software captures the date, start time and duration of the demonstration. It also determines the geolocation coordinates of an SR using GPS surveillance. After meetings, each SR submits an activity report, setting several additional parameters:
- type of activity (e.g. individual, group, remote);

- types of a customer in the workplace and specialty, concerning the product or service being promoted;

- recommended number of activities with this customer.

Therefore, the specificity of the work done by SRs is that their activities cannot be tracked by an employer, and values of additional parameters in the activity reports are the private opinion of an SR.

On the other hand, there are well-controlled feedback options, such as profit, sales volume, numbers of contracts, transactions and new customers; execution of plan performance indicators are irrelevant for SR only promoting products or services. Thus, because of the inability to control the results, an employer is forced to move to control the process - those actions that are committed to achieving the goal. This includes the employee's compliance with the company's regulations, quality of ser- 
vice, and communication with customers, knowledge of a product, ability to make their own decisions. But the question of evaluating the productivity of SRs remains open.

\section{State of the art}

According to the data of Dimensions.ai [1], which provides access to worldwide information about the results on grants, patents, publications, and other sources, the number of publications and patents in the field of evaluating the productivity of managers using modern data analysis methods is increasing from year to year (Figure 1). Some decline in 2020 was caused by a one-two-year period (on average) that lasts from submission of an article or an application to its publication or issue of a patent.

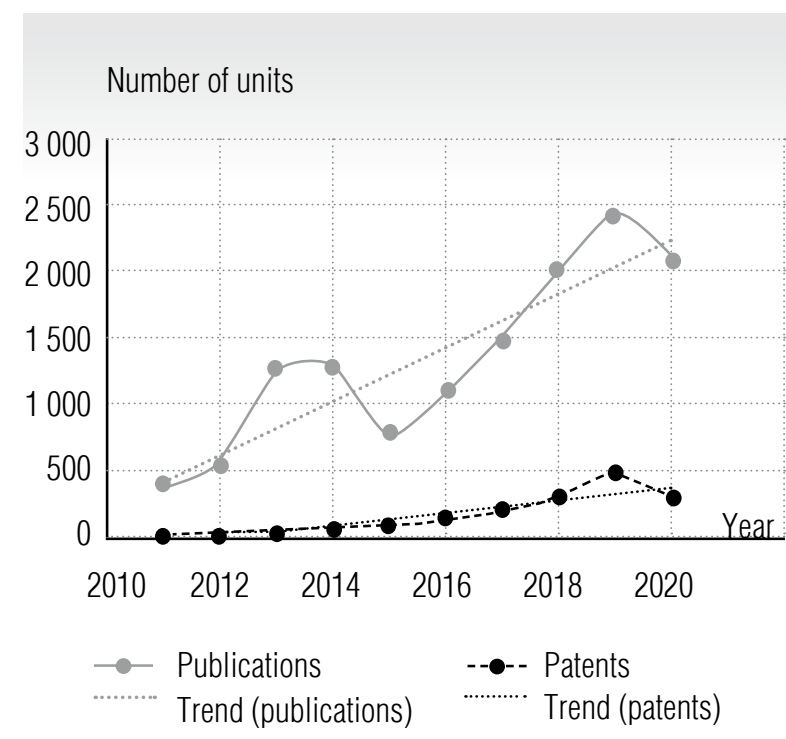

Fig. 1. Trends in the number of publications and patents

Existing studies evaluating the productivity of SRs, as well as identifying the dependencies between controlled parameters and performance of employees are mainly based on analysis of standard methods of management, calculations of numerical performance indicators, and usage of analytical and CRM systems.
The article [2] assesses the performance of sales management approaches in three companies. An investigation was made into the validity of distribution of tasks and products between managers, the validity of focusing on certain territories and planning product presentations. Information for the study was obtained during interviews with sales and marketing managers, monitoring the medical representatives' activities, and studying the organization's reports and market data.

The study [3] identifies performance control methods such as telephone calls, external and internal audits, double visits which assess the professional and communication skills of an employee, ability to present a product, daily reports in the CRM system, and their evaluation, as well as evaluation of financial reports.

The article [4] insists on intelligent planning of SR's activities, since this staff is one of the most expensive in pay and training. It was revealed that the SR's work is deeply digitized, because CRM-systems fix many parameters: from time in transport to the number of meetings per month. Existing analytical solutions build routes to the clients based on travel time, possible cancellation of a meeting and other parameters, allowing us to determine the optimal sequence of visiting clients. At the same time, a comprehensive analysis of the data collected does not always work properly. Moreover, the key parameter of SR performance, the human factor, is difficult to analyze.

The authors of [5] looked at the digital technologies applied in the health care field. The proposed measures are related to the implementation of KPI monitoring systems to assess the activities of health care workers (e.g., the result of a consultation, a telephone conversation of a clinic administrator). Using these systems, it is possible to determine a department that works most effectively, a service that brings the most profit.

The article [6] describes an application of a hybrid procedure based on the K-mean and the tree decision methods to predict employee per- 
formance for the next year. It operates with factors such as personality, punctuality, eloquence, etc. The algorithm predicts the number of employees selected to be promoted or fired and helps to identify ineffective employees.

The article [7] is dedicated to describing human behavior as a chain of mathematical models (Kalman filters, ordered together by the Markov chain). These models are used to recognize a person's sensory behavior and predict his actions (e.g., follow-up of drivers based on preparatory movements). In the article, the authors applied a naive Bayesian classifier to a set of three years of sales data from a global transportation company. The classification was conducted within three classes: fails to cope with responsibilities, copes, and shows outstanding results. The authors propose to use a naive Bayesian classifier to evaluate the work of SRs using as much information from CRM systems as possible.

The paper [8] says that KPIs are formed based on fragmented knowledge about business processes. The authors of the article presented a method that allows us to validate KPI properties before implementation and to combine target and conceptual modeling.

Thus, our literature analysis showed that along with a high level of digitizing business processes, the SR's activity in most cases is still being evaluated by surveys, opinions of experts and costly double visits. At the same time, the presence of a large sample of data allows us to use statistical analysis to identify both insufficient and inflated performance indicators of an SR activity.

\section{Source data}

The source data is accumulated by a B2Bdistributor of drugs and BADs. The company has two business units (RX and OTC) and five divisions (T1, T2, T3, T4, T5). Marketing activities of the company are focused on familiarizing decision-makers with the characteristics of products (within the framework of promotions). Parameters of meetings and parameters from SRs meeting reports are sent into a distributed database run by CRM. It keeps records of SRs, customers, products and services. The available sample contains more than three hundred thousand observations on 28 different features, which include:

- unique digital and/or symbolic IDs of SRs and their activities, IDs of clients and their companies, as well as promotions within an activity;

- categorical features of activity and company types, specialty, division, category and the target group of customers, promotional campaign, flags of the activity performance, of the presence of an SR at an activity;

- temporary features of beginning and end of an activity, duration of a presentation, an agreement on the brand, time of determining coordinates;

- integer features (number of meetings, results of promotions, flags);

- real and geolocation features (longitude and latitude of the activity's place).

Thus, there are moderately heterogeneous digital data with a predominance of service information (Figure 2a). Because of the data omissions, some types have not been identified correctly. After data type conversion (as explicit conversion, as well as determining the time of a day, the day of a week, the season of a year by the date of the meeting), the ratio of the integer, real, time, and categorical features has strengthened in favor of integer and categorical features (Figure 2b).

\section{Technique of statistical analysis}

Traditionally, there are three stages of statistical study $[9,10]$ : 

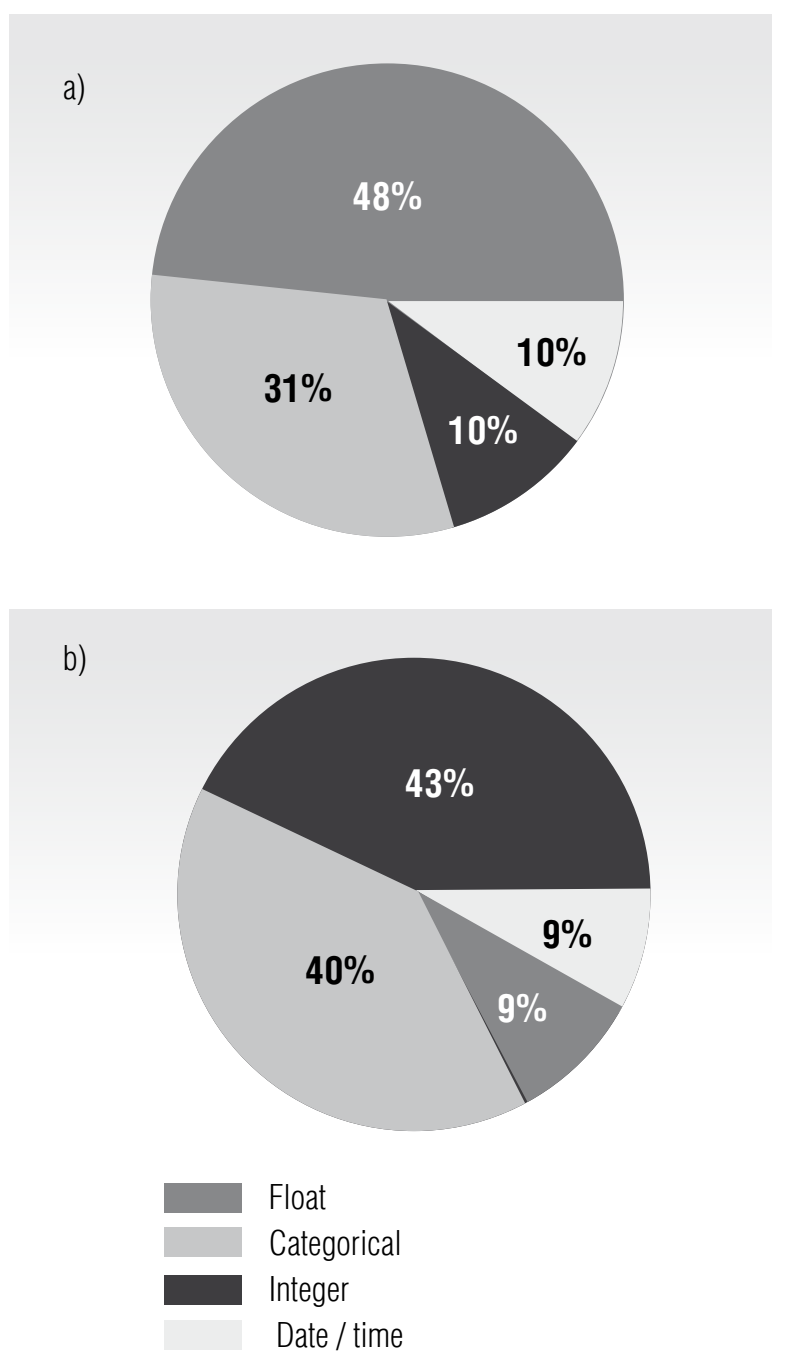

Fig. 2. The ratio of the data types:

(a) before the conversion; b) after the conversion

- the collection and processing of primary data;

- a statistical summary and a grouping of processed information;

- setting statistical hypotheses and interpreting the results.

Table 1 details the main stages of the study using approaches proposed by data mining [11, $12]$ and machine learning $[13,14]$.
The following are the main results from applying the proposed technique of statistical analysis.

\section{Stage 1: \\ Collecting and processing primary data}

\subsection{Data audit}

Time series modeling suggests the investigation of the relationship between the values of features in time. Using a special type of scatter plot - a lag plot [15], we studied autocorrelation dependencies of two features with the defined observation lag (Figure 3).

A greater number of points in the diagonal area suggests a stronger autocorrelation dependency. Points concentrated in the middle or distributed throughout the area of the figure suggest weak dependency. The undetectable chaotic structure of the chart points indicates that the data is random.

The results show the presence of a structure in values of both categorical features that speak of their non-randomness. The existence of autocorrelation dependencies is emphasized by a second diagonal passing through the upper right and lower left corners. It has been established that for big data this type of plot is built much faster than the classic autocorrelation one.

\subsection{Synthesis of time and geolocation features}

According to the time features, storing moments of the beginning and end of presentations, we synthesized an integer feature the number of a day in a year and categorical features: the day of a week; the season of a year and the period of a day when the activity was carried out. According to geolocation features of latitude and longitude, we built a map of business units activity (Figure 4) in Tableau ${ }^{1}$ software, and created synthetic features (the postcode, the address, and the region) $[16,17]$.

\footnotetext{
${ }^{1}$ http://elearning.tableau.com/
} 
Stages of statistical analysis

Table 1.

\begin{tabular}{|c|c|c|}
\hline \multirow{4}{*}{1.} & \multirow{4}{*}{$\begin{array}{l}\text { Collecting and processing } \\
\text { of primary data }\end{array}$} & Data audit, outliers and omissions processing \\
\hline & & Typification and encoding features \\
\hline & & Synthesis of time and geolocation features \\
\hline & & Building a tag cloud \\
\hline \multirow{4}{*}{2.} & \multirow{4}{*}{$\begin{array}{l}\text { Summarizing and grouping } \\
\text { the information proessed }\end{array}$} & Profiling features \\
\hline & & Selecting the type and parameters of the features distribution \\
\hline & & Selecting weakly correlated features \\
\hline & & Grouping data \\
\hline \multirow{3}{*}{3.} & \multirow{3}{*}{$\begin{array}{l}\text { Setting hypotheses and } \\
\text { interpretation of results }\end{array}$} & Setting a statistical hypothesis \\
\hline & & Modeling \\
\hline & & Interpretation of results \\
\hline
\end{tabular}

ID of sales representatives with a lag

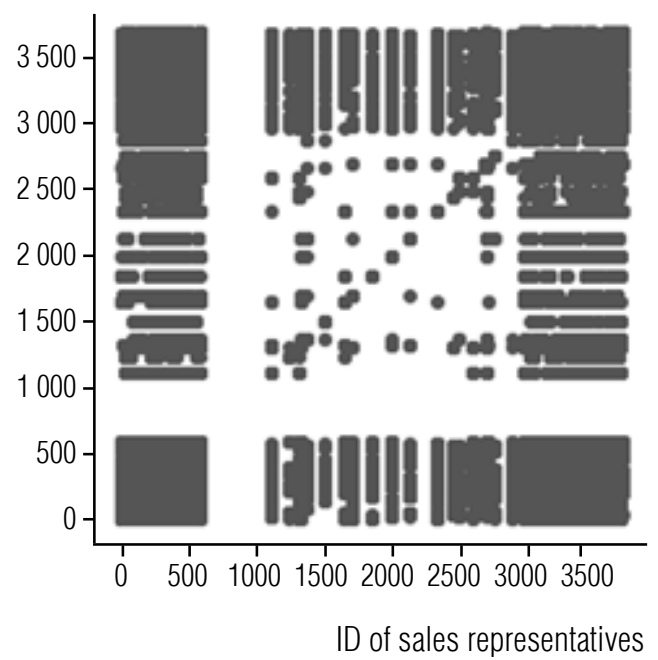

ID of activities with a lag

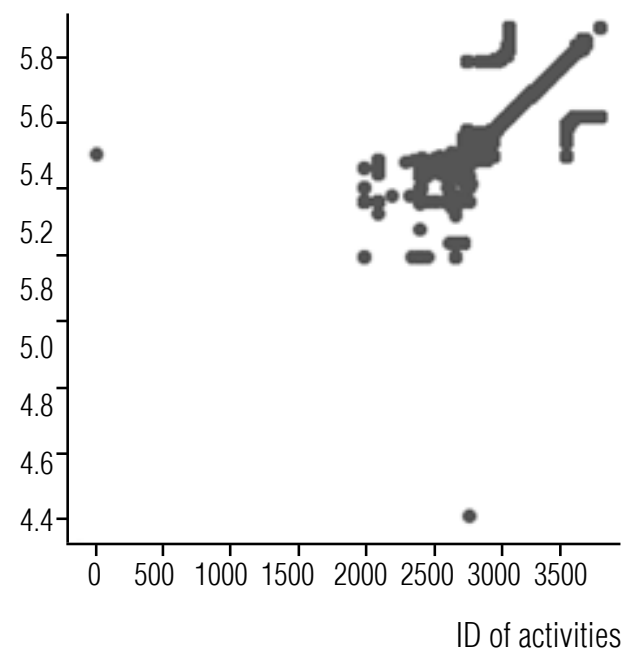

Fig. 3. Non-randomness of feature's data:

(a) identifiers of SRs, lag is 30 observations; (b) activity identifiers, lag of 50 observations

Based on mapping data, it can be concluded that, for example, most of the activities are carried out by the OTC unit. It also exclusively works on the territory of the Sakhalin and Kaliningrad regions.

\subsection{Tag cloud design}

To analyze values of 12 categorical and temporal features converted to categorical, a tag cloud was built, using the WordCloud ${ }^{2}$ library of the Python language (Figure 5).

\footnotetext{
${ }^{2}$ https://python-graph-gallery.com/wordcloud/
} 


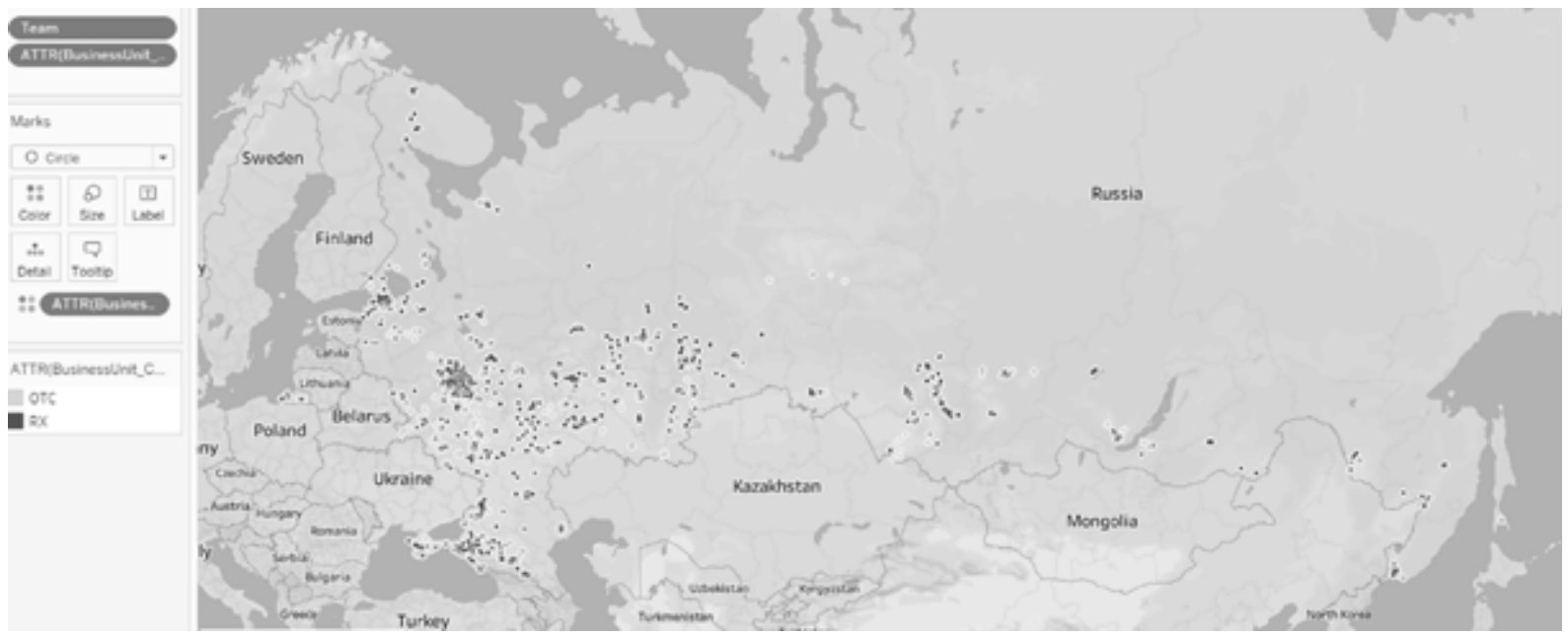

Fig. 4. Activity map of two business units

Using the tag cloud, we selected:

- the most popular season for promotional campaigns is winter (autumn and spring seasons go with a significant lag);

- the most popular meeting time with clients is afternoon (morning time takes second place);

- the most productive business unit by activity number is RX;

- the preferred type of communication with clients is the visit (consultancy takes second place);

- the days of the week with the highest number of contacts with clients are Tuesday and

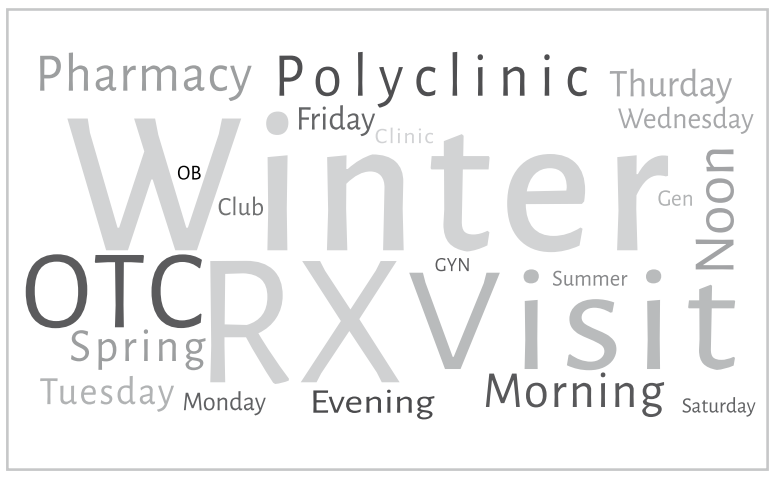

Fig. 5. Tag cloud of categorical features
Thursday (in second place - Wednesday and Friday). A significant number of records of Sunday meetings have been established;

- the most frequent clients are polyclinics and pharmacies.

\section{Summarizing statistics and grouping of information processed}

\subsection{Profiling features}

Using the library named pandas-profiling, we evaluated the relationship of features and built histograms of feature distributions [18-20]. Figure 6 shows the frequency of activities across two business units of the company according to client types.

The most interesting is close to the normal distribution of the ID of activity (Figure 7a). This random value successfully passes the normality test "normaltest" from the SciPy library, which combines tests of D'Agostyno and Pearson on excess and an asymmetry [21], if we reduce tails, removing outliers (Figure $7 b$ ).

We convert the normal distribution of the ID of activity to the standard normal distribution $X_{n}$ by the formula: 

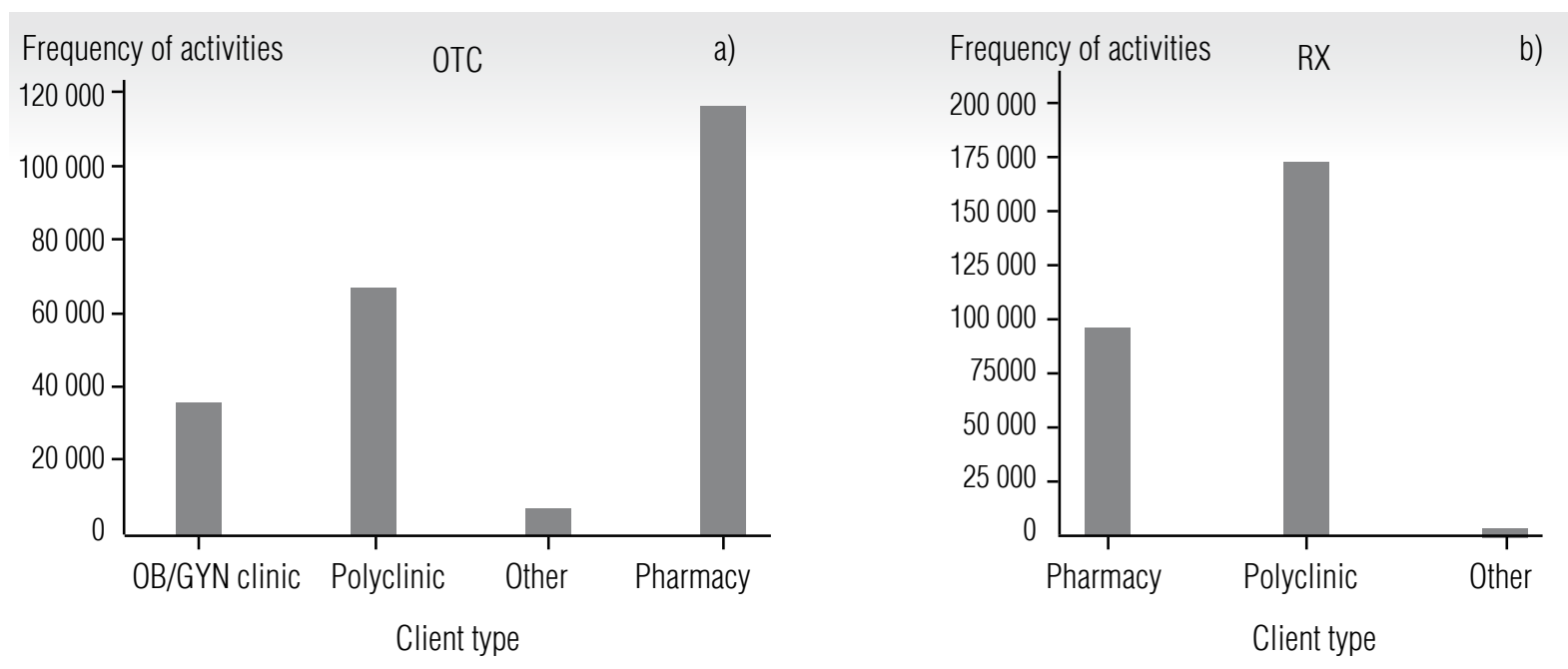

Fig. 6. Distribution of the frequency of activities with different types of clients into two business units: a) OTC; b) RX
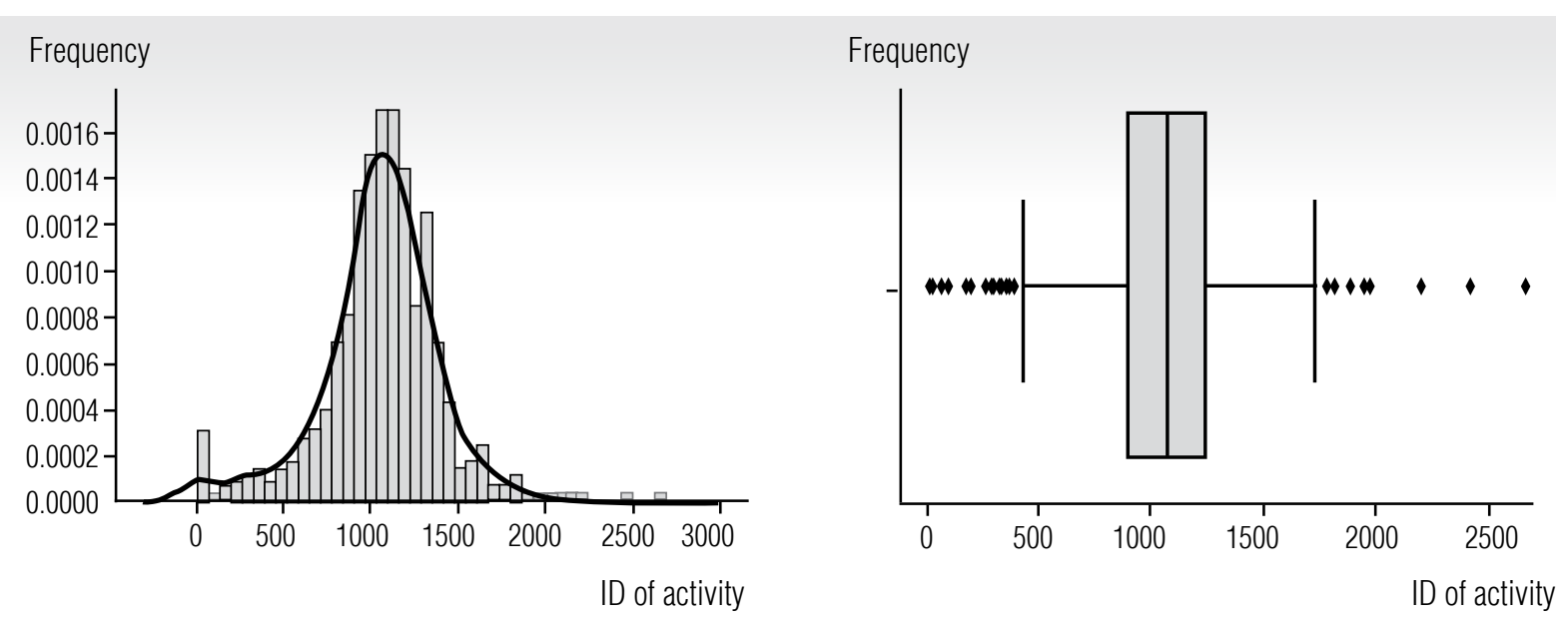

Fig. 7. Distribution of the ID of activity:

a) Iong right tail; b) outliers

$$
X_{n} \sim \operatorname{Norm}(0 ; 1)=\frac{X-m_{x}}{\sigma_{x}}
$$

where $m_{x}-$ a mean of the ID of activity;

$\sigma_{x}-$ a standard deviation of the ID of activity.

The distribution of this feature allowed us to formulate a statistical hypothesis evaluating the level of distortion of the activity parameters.

\section{Hypotheses and interpretation of results}

To model the level of distortion of activity parameters, we use a probabilistic approach [22-24]. Let $H_{1}$ is a hypothesis that an activity was carried out, and the alternative hypothesis $\mathrm{H}_{2}$ means that the activity was not carried out. Let the event $A$ - hit the parameters of the conducted or not conducted activity in the database. Then by the formula of total probability 
the probability of event $A$ is calculated as follows:

$$
P(A)=P\left(H_{1}\right) P\left(A \mid H_{1}\right)+P\left(H_{2}\right) P\left(A \mid H_{2}\right)
$$

Let the level of the normalized ID of activity $X_{n}$ of a certain SR be $p$. Let the probability that this SR inputs parameters of a failed activity into the database be defined as $P_{m}$. Then, given (1), rewrite (2) as follows:

$$
P(A)=p \cdot 1+(1-p) P_{m}
$$

Since there are many SRs who input information about meetings in the distributed database, we deal with a flow of information. For the initial model's settings, it is desirable to know a portrait of an average SR, namely the level of his $p$. Let us say the most common situation is when the normalized ID of activity of an SR equals 0.5. Let the normally distributed random value $p \sim N(0.5 ; 0.1)$ model the input flow. It is clear that an SR cannot radically change the parameters of his activities, otherwise, they will become outliers. Let an SR change the normalized ID of activity by $s$ times, $s \in[0 ; 1]$ concerning the average value, raising it to $p^{\prime}$ :

$$
p^{\prime}=p+(1-p) s
$$

As a result, with a probability $P_{m}=(1-p) s$ an SR distorts the data, and with a probability of $1-P_{m}=p$ inputs undistorted data. Figure 8 shows the modeling results in the case when SRs distort the information about activities by $10 \%$.

Further analysis is aimed at confirming the hypothesis that based on the existing data set it is possible to identify SRs whose input information is knocked out of the sample. Note that it is also possible to make predictions on known models considering the timebased features [25].

\section{Discussion}

Initial features are grouped by the ID of SRs and we consider all observations related to a particular SR. At the same time, several patterns were identified to generate features by their semantic essence [26, 27]: numerical features denoting an amount were summarized, and their average and median were also defined. Among these features are the duration of a presentation, an agreement on the brand, time of determining the coordinates of an SR. For the features that are identifiers, we generated a feature of the number of unique values that were found among observations on an SR [28].

Thus, the new data (one observation - one $\mathrm{SR}$ ) are obtained and it's now possible to select the SRs whose numbers of numerical features are outliers (Figure 9).

It should be noted that the proposed approach based on the grouping of data and the passing of the statistical test is most effective for dropping out highly distorted parameters and is much less effective for identifying poorly distorted parameters. Therefore, at this stage of the study, before the development of a statistical test system or a separate mathematical model for identifying poorly distorted parameters, we propose to evaluate the KPI of SRs on an integral characteristic that takes into account both the results obtained under the proposed approach and peer assessments.

\section{Conclusion}

As a result of data mining, we set up a statistical hypothesis about the possibility of identifying sale representatives who distort the number and parameters of their meetings with clients. A set of synthetic integer, real and categorical features has been created to identify hidden relationships in the given dataset. Doubtful data (such as working hours 


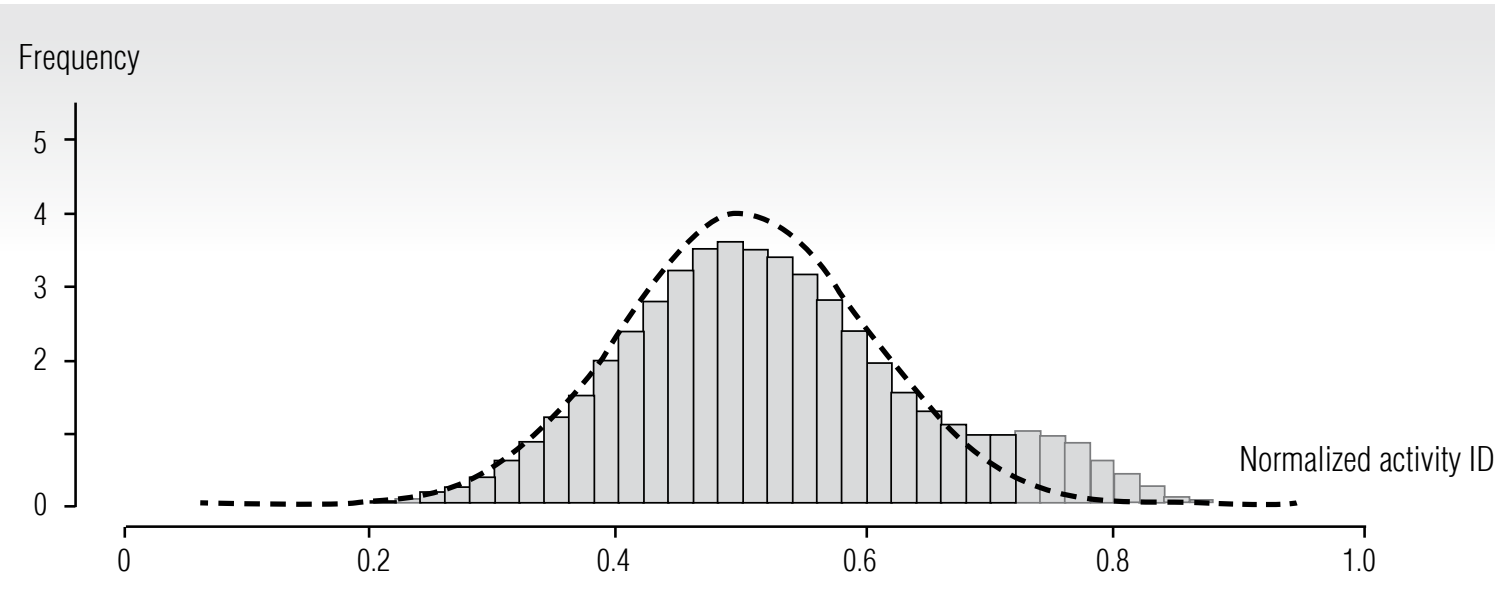

Fig. 8. The result of distortion of $10 \%$ of activity parameters
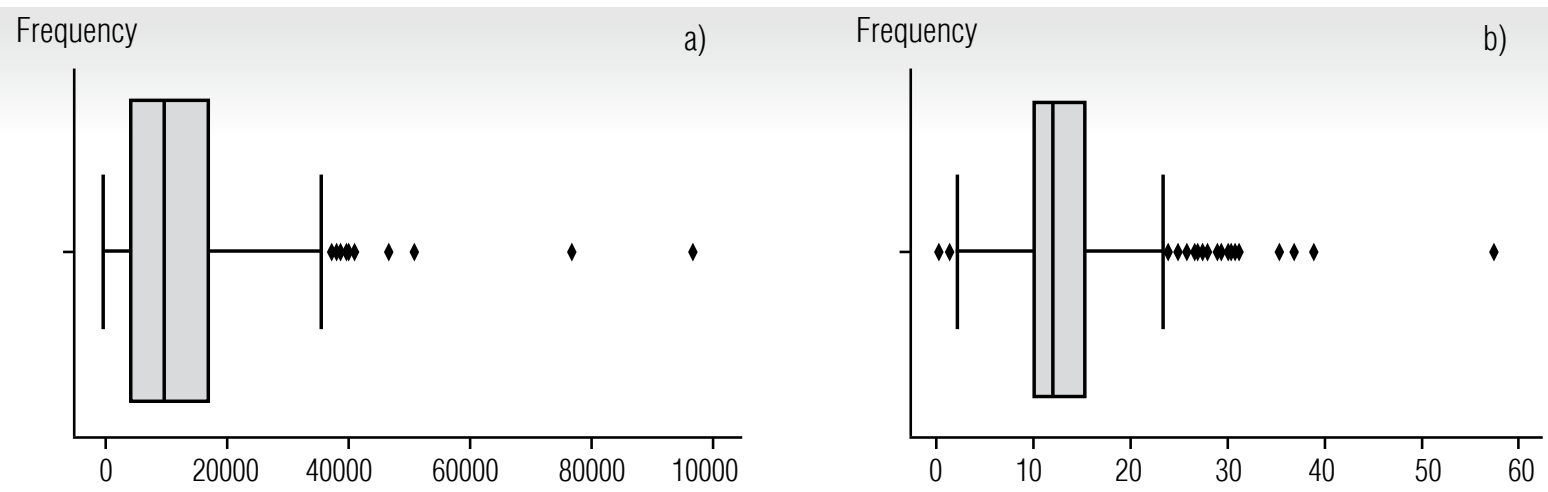

Fig. 9. Feature outliers: a) the brand agreement, units; b) duration of activity, days

at weekends and nights) have been revealed. The resulting aggregated dataset is grouped by the ID of activity and its distribution was built. A probabilistic simulation of the activity of SRs was carried out to assess the level of information distortion. For each sale repre- sentative, the integer, real and categorical features are summarized and outliers that characterize inefficient productivity or distortion of data have been detected. A combination of statistical and expert assessments is proposed to reduce type II error.

\section{References}

1. Bode C., Herzog C., Hook D., McGrath R. (2018) Dimensions Report. Cambridge, MA: Digital Science.

2. Dimitriadi N.A. (2007) Efficiency of an entrepreneurial project: audit of sales management systems. Accounting and Statistics, no 2, pp. 142-147 (in Russian).

3. Baeva O.N., Khomyakova S.G. (2015) Managing remote workers: the experience of pharmaceutical companies. Baikal Research Journal, vol. 6, no 5, pp. 7. DOI: 10.17150/2411-6262.2015.6(5).18 (in Russian). 
4. Baranov R.A. (2018) Intelligent planning of the work of medical representatives. Digital Economy. Available at: https://www.comnews.ru/digital-economy/content/116565/2018-12-10/intellektualnoeplanirovanie-raboty-medicinskih-predstaviteley (accessed 22 November 2020) (in Russian).

5. Iljashenko O., Bagaeva I., Levina A. (2019) Strategy for establishment of personnel KPI at health care organization digital transformation. IOP Conference Series: Materials Science and Engineering, no 497, article ID 012029. DOI: 10.1088/1757-899X/497/1/012029.

6. Sarker A., Shamim S.M., Zaman Md.S., Rahman Md.M. (2018) Employee's performance analysis and prediction using k-means clustering \& decision tree algorithm. Global Journal of Computer Science and Technology: Software and Data Engineering, vol. 18, no 1, pp. 1-6.

7. Pentland A., Liu A. (1999) Modeling and prediction of human behavior. Neural Computation, vol. 11, no 1, pp. 229-242.

8. Roubtsova E., Michell V. (2013) A method for modeling of KPIs enabling validation of their properties. Proceedings of the 5th ACM SIGCHI Annual International Workshop on Behaviour Modelling - Foundations and Applications. Montpellier, France, 2 July 2013, article ID 3. DOI: 10.1145/2492437.2492440.

9. Calixto N., Ferreira J. (2020) Salespeople performance evaluation with predictive analytics in B2B. Applied Sciences, vol. 10, no 11, article ID 4036. DOI: 10.3390/app10114036.

10. Vladova A.Yu. (2018) Clustering analysis of changes in the spatial position of the trunk oil pipeline sections based on the in-line inspection datasets. Occupational Safety in Industry, no 1, pp. 22-25 (in Russian).

11. Larose D.T., Larose C.D. (2015) Data mining and predictive analytics. Wiley.

12. Leskovec J., Rajaraman A., Ullman J.D. (2014) Mining of massive datasets. Stanford, CA: Stanford InfoLab.

13. VanderPlas J. (2016) Python data science handbook. O'Reilly Media.

14. Joshi P. (2017) Artificial intelligence with Python. Packt.

15. Brownlee J. (2017) Introduction to time series forecasting with Python: how to prepare data and develop models to predict the future. Machine Learning Mastery.

16. Ko I., Chang H. (2017) Interactive visualization of healthcare data using Tableau. Healthcare Informatics Research, vol. 23, no 4, pp. 349-354. DOI: 10.4258/hir.2017.23.4.349.

17. Audric S., De Bellefon M.-P., Durieux E. (2018) Descriptive spatial analysis. Handbook of spatial analysis. Theory and practical application with $R$. Insee Methodes, no 131, pp. 3-30.

18. Brink H., Richards J.W., Fetherolf M. (2016) Real-world machine learning. Manning.

19. Ismail A. (2020) How to use Pandas-Profiling on Google Colab. Python in Plain English. Available at: https://python.plainenglish.io/how-to-use-pandas-profiling-on-google-colab-e $34 \mathrm{f} 34 \mathrm{ff} 1 \mathrm{c} 9 \mathrm{f}$ (accessed 31 October 2020).

20. Skiena S.S. (2017) The data science design manual. Springer.

21. Vadzinsky R. (2018) Statistical calculations in the Excel environment. Saint Petersburg: Piter (in Russian).

22. Brownlee J. (2020) Statistical methods for machine learning. Machine Learning Mastery.

23. Denezhkina I.E., Zadadaev S.A. (2018) Testing statistical hypotheses using visualization tools in the R Studio environment. Proceedings of the V International Scientific and Practical Conference "System Analysis in Economics”. Moscow, Russia, 21-23 November 2018, pp. 181-184 (in Russian).

24. Soloviev V.I. (2019) Data analysis in economics: probability theory, applied statistics, data processing and visualization in Microsoft Excel. Moscow: KnoRus (in Russian).

25. Lukashin Yu.P. (2003) Adaptive methods of short-term time series forecasting. Moscow: Finance and Statistics (in Russian).

26. Flach P. (2012) Machine learning: The art and science of algorithms that make sense of data. Cambridge: Cambridge University Press.

27. McKinney W. (2012) Python for data analysis: Data wrangling with Pandas, NumPy, and IPython. O'Reilly Media. 
28. Vladova A.Yu., Vladov Yu.R. (2020) Digitalization of marketing campaigns. Proceedings of the 2-nd International Scientific and Practical Conference "Digital Transformation of Industry: Trends, Management, Strategies - 2020”. Ekaterinburg, Russia, 27 November 2020, pp. 67-74 (in Russian).

\section{About the authors}

\section{Alla Yu. Vladova}

Dr. Sci. (Tech.);

Leading Researcher, V.A. Trapeznikov Institute of Control Sciences, Russian Academy of Sciences, 65, Profsoyuznaya Street, Moscow 117997, Russia;

Professor, Department of Mathematics, Financial University under the Government of the Russian Federation, 49, Leningradsky Prospect, Moscow 125993, Russia;

E-mail: ayvladova@fa.ru

ORCID: 0000-0002-8556-3798

\section{Elena D. Shek}

Student, Plekhanov Russian University of Economics, 36, Stremyanny Lane, Moscow 117997, Russia;

E-mail: 1399selena@gmail.com 